\title{
The male and female complete mitochondrial genome sequences of the Endangered freshwater mussel Potomida littoralis (Cuvier, 1798) (Bivalvia: Unionidae)
}

Elsa Froufe, Han Ming Gan, Yin Peng Lee, João Carneiro, Simone Varandas, Amílcar Teixeira, Alexandra Zieritz, Ronaldo Sousa \& Manuel Lopes-Lima

To cite this article: Elsa Froufe, Han Ming Gan, Yin Peng Lee, João Carneiro, Simone Varandas, Amílcar Teixeira, Alexandra Zieritz, Ronaldo Sousa \& Manuel Lopes-Lima (2016) The male and female complete mitochondrial genome sequences of the Endangered freshwater mussel Potomida littoralis (Cuvier, 1798) (Bivalvia: Unionidae), Mitochondrial DNA Part A, 27:5, 3571-3572, DOI: 10.3109/19401736.2015.1074223

To link to this article: https://doi.org/10.3109/19401736.2015.1074223

册 Published online: 14 Aug 2015.

џ Article views: 86
Submit your article to this journal $\pi$ 


\title{
The male and female complete mitochondrial genome sequences of the Endangered freshwater mussel Potomida littoralis (Cuvier, 1798) (Bivalvia: Unionidae)
}

\author{
Elsa Froufe ${ }^{1}$, Han Ming Gan ${ }^{2,3}$, Yin Peng Lee ${ }^{2,3}$, João Carneiro ${ }^{1}$, Simone Varandas ${ }^{4}$, Amílcar Teixeira ${ }^{5}$, \\ Alexandra Zieritz ${ }^{6}$, Ronaldo Sousa ${ }^{7}$, and Manuel Lopes-Lima ${ }^{1}$ \\ ${ }^{1}$ CIIMAR/CIMAR - Interdisciplinary Centre of Marine and Environmental Research, University of Porto, Porto, Portugal, ${ }^{2}$ School of Science, Monash \\ University Malaysia, Petaling Jaya, Selangor, Malaysia, ${ }^{3}$ Monash University Malaysia Genomics Facility, Monash University Malaysia, Petaling Jaya, \\ Selangor, Malaysia, ${ }^{4}$ CITAB-UTAD - Centre for Research and Technology of Agro-Environment and Biological Sciences, University of Trás-os-Montes \\ and Alto Douro, Vila Real, Portugal, ${ }^{5}$ CIMO-ESA-IPB - Mountain Research Centre, School of Agriculture, Polytechnic Institute of Bragança, \\ Campus de Santa Apolónia, Bragança, Portugal, ${ }^{6}$ Faculty of Science, School of Geography, University of Nottingham Malaysia Campus, Semenyih, \\ Selangor Darul Ehsan, Malaysia, and ${ }^{7} C B M A$ - Centre of Molecular and Environmental Biology, Department of Biology, University of Minho, \\ Campos de Gualtar, Braga, Portugal
}

\section{Abstract}

Freshwater mussels of the family Unionidae exhibit a particular form of mitochondria inheritance called double uniparental inheritance (DUI), in which the mitochondria are inherited by both male and female parents. The (M)ale and (F)emale mitogenomes are highly divergent within species. In the present study, we determine and describe the complete $M$ and $F$ mitogenomes of the Endangered freshwater mussel Potomida littoralis (Cuvier, 1798). The complete $\mathrm{M}$ and $\mathrm{F}$ mitogenomes sequences are $16451 \mathrm{bp}$ and $15787 \mathrm{bp}$ in length, respectively. Both $F$ and $M$ have the same gene content: 13 protein-coding genes (PCGs), 22 transfer RNA (trn) and 2 ribosomal RNA (rrn) genes. Bayesian analyses based on the concatenated nucleotide sequences of 12 PCGs and 2 rrn genes of both genomes, including mitogenome sequences available from related species, were performed. Male and Female lineages are monophyletic within the family, but reveal distinct phylogenetic relationships.
\end{abstract}

\section{Keywords}

Ambleminae, Anodontinae, Gonideinae, mitogenome, phylogeny

\section{History}

Received 9 July 2015

Accepted 9 July 2015

Published online 14 August 2015
The freshwater mussel Potomida littoralis (Bivalvia: Unionidae) is an Endangered freshwater mussel with a circum-Mediterranean distribution (Lopes-Lima et al., 2014). Phylogenetic studies within the family are scarce with descriptions of several subfamilies being based primarily on morphological traits (Carter et al., 2011). The inclusion of many genera in these subfamilies is still unclear, mostly due to the lack of sampling coverage and number of available markers (Bogan, 2008). Unionid bivalves, such as several other bivalve families, possess an interesting mitochondrial inheritance, called doubly uniparental inheritance (DUI), in which the male gonad tissue contains (M) mitochondria inherited from the fathers, the male somatic tissue contains (F) mitochondria inherited from the mothers, and females inherit their (F) mitochondria through ordinary single uniparental inheritance (Hoeh et al., 1996). The $\mathrm{M}$ and $\mathrm{F}$ mitochondrial lineages are highly divergent within each animal and have distinct gene order arrangements (Huang et al., 2013).

In this study, the complete $\mathrm{M}$ and $\mathrm{F}$ mitogenomes of $P$. littoralis were sequenced, assembled and annotated using an established pipeline (Gan et al., 2014). The M and F mitogenomes have been deposited in GenBank database under the accession

Correspondence: Elsa Froufe, CIIMAR/CIMAR - Interdisciplinary Centre of Marine and Environmental Research, University of Porto, Rua dos Bragas 289, 4050-123 Porto, Portugal. E-mail: elsafroufe@gmail.com numbers KT247375 and KT247374, respectively. The length of the $\mathrm{F}$ and $\mathrm{M}$ mitogenomes (16 $451 \mathrm{bp}$ and $15787 \mathrm{bp}$, respectively) of $P$. littoralis sequenced in this study is within the expected range for each gender-specific haplotypes within Unionidae. This is mainly due to the $3^{\prime}$ extension of $\mathrm{COX} 2$, common to all freshwater mussel male mitogenomes. Both haplotypes have the same gene content: 13 protein-coding genes (PCGs), 22 transfer RNA (trn) and 2 ribosomal RNA (rrn) genes involved in mitochondrial transcription and translation processes. Regarding the gene orientation, again both have the same genes (4 PCGs, 20 tRNAs and 2 rRNAs) encoded on the heavy strand and the remaining (9 PCGs and 2 tRNAs) encoded on the complementary strand.

Additional mitogenome sequences ( $\mathrm{M}$ and $\mathrm{F}$ ) available from related species were downloaded from GenBank. Each gene sequence was aligned using GUIDANCE (version 1.5, Penn et al., 2010) with the MAFFT multiple sequence alignment algorithm (version 7, Katoh \& Standley, 2013). To build our single gene alignments, we used the following GUIDANCE parameters: score algorithm: GUIDANCE; bootstraps replicates: 100; Sequence cutoff score: 0.0 (no sequences removed); Column cut-off score: below 0.8. The final concatenated data set included 12 mitochondrial protein-coding genes (excluding the ATP8 due to its extreme length variation) and the 2 rRNA genes of both genomes. To infer the phylogenetic relationships among these sequences with Bayesian methodology, we used MrBayes v3.2.1 (Ronquist et al., 2012). The alignment (12084 bp) was partitioned according to the best scheme determined by JmodelTest2 


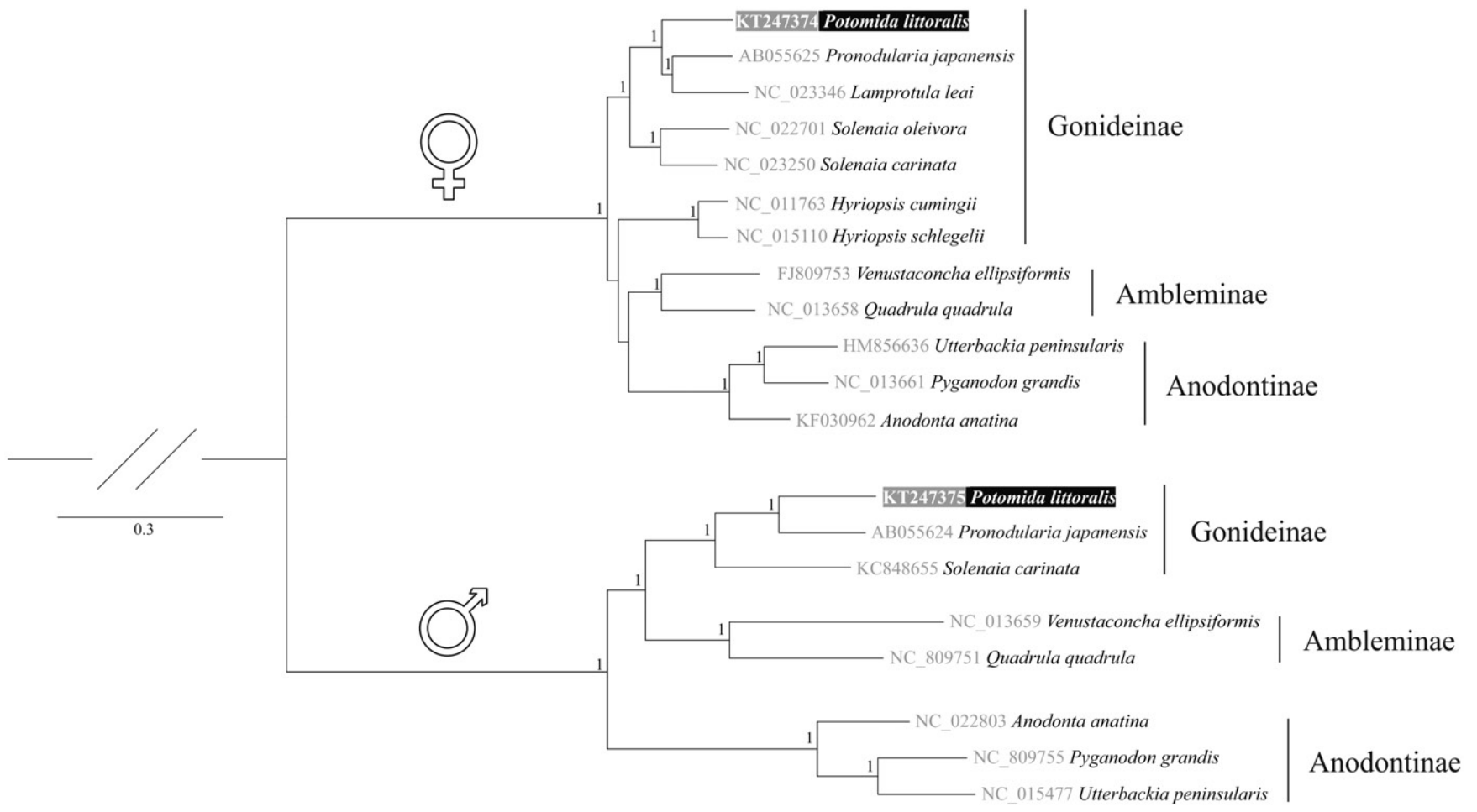

Figure 1. Bayesian phylogenetic tree of 20 Unionidae Male and Female mitogenomes sequences based on concatenated nucleotide sequences of 12 mitochondrial protein-coding genes (excluding the ATP8 gene) and the 2 rRNA genes. The numbers behind the species names are the GenBank accession numbers and those at the nodes indicate the posterior probability.

$(\mathrm{GTR}+\mathrm{I}+\mathrm{G}, \mathrm{HKY}+\mathrm{I}+\mathrm{G}$ and $\mathrm{HKY}+\mathrm{G}$ models were used $)$. Each chain started with a randomly generated tree and ran for $1 \times 10^{6}$ generations with a sampling frequency of 1 tree for every 100 generations. The resultant trees, after discarding the first $25 \%$ as burn-in, were combined in a 50\% majority rule consensus tree. The final BI tree was rooted at the split between Male and Female haplotypes (based on previous studies, e.g. Huang et al., 2013).

As expected, the obtained phylogenetic tree (Figure 1) revealed two main clades divided between the monophyletic $\mathrm{M}$ and $\mathrm{F}$ mitogenomes clades. However, the three sub-families (for which GenBank sequences are available) revealed distinct phylogenetic relationships. Although a smaller number of $\mathrm{M}$ genomes are available, relationships are very well resolved and supported only for $\mathrm{M}$ genomes but not for $\mathrm{F}$ genomes. In the $\mathrm{M}$ clade, the Gonideinae and Ambleminae form a sister lineage and then sister to the Anodontinae, whereas in the F-clade, the relationships of these subfamilies are not well resolved. The newly sequenced $P$. littoralis genomes cluster inside the Gonideinae with Pronodularia japanensis in the M-clade, and with a clade including P. japanensis and Lamprotula leai in the F-clade.

\section{Declaration of interest}

The authors report that they have no conflicts of interest. The authors alone are responsible for the content and writing of the paper. This work was financially supported by the Portuguese Foundation for Science and Technology (FCT) project PTDC/AAC-AMB/ 117688/2010, and partly by the Strategic Funding UID/Multi/04423/2013 through national funds provided by FCT and European Regional Development Fund (ERDF), in the framework of the programme PT2020. JC is supported by Portuguese Foundation for Science and Technology (FCT) post-doc fellowship with reference SFRH/BPD/100912/2014. HMG and YPL are supported by the Monash University Malaysia Tropical Medicine Biology Platform.

\section{References}

Bogan AE. (2008). Global diversity of freshwater mussels (Mollusca, Bivalvia) in freshwater. Hydrobiologia 595:139-47.

Carter JG, Altaba CR, Anderson LC, Araujo R., et al. (2011). A synoptical classification of the Bivalvia (Mollusca). Paleont Contr 4:1-47.

Gan HM, Schultz MB, Austin CM. (2014). Integrated shotgun sequencing and bioinformatics pipeline allows ultra-fast mitogenome recovery and confirms substantial gene rearrangements in Australian freshwater crayfishes. BMC Evol Biol 14:19.

Hoeh WR, Stewart DT, Sutherland GW, Zouros E. (1996). Multiple origins of gender-associated mitochondrial DNA lineages in bivalves (Mollusca: Bivalvia). Evol 50:2276-86.

Huang XC, Rong J, Liu Y, Zhang MH, et al. (2013). The complete maternally and paternally inherited mitochondrial genomes of the endangered freshwater mussel Solenaia carinatus (Bivalvia: Unionidae) and implications for Unionidae taxonomy. PloS One 8:e84352.

Katoh K, Standley DM. (2013). MAFFT multiple sequence alignment software version7: Improvements in performance and usability. Mol Biol Evol 30:772-80.

Lopes-Lima M, Prie V, Seddon MB. (2014). Potomida littoralis. The IUCN Red List of Threatened Species. Version 2014.2. Available at: www.iucnredlist.org

Penn O, Privman E, Ashkenazy H, Landan G, Graur D, Pupko T. (2010) GUIDANCE: a web server for assessing alignment confidence scores. Nucleic Acids Res 38 (Web Server issue):W23-W28.

Ronquist F, Teslenko M, Van Der Mark P, Ayres DL, Darling A, Hna S, Larget B, et al. (2012). MrBayes 3.2: Efficient Bayesian phylogenetic inference and model choice across a large model space. Syst Biol 61: $539-42$. 